\title{
Cannabinoid type 1 receptors in human skeletal muscle cells participate in the negative crosstalk between fat and muscle
}

\author{
K. Eckardt $\cdot$ H. Sell $\cdot$ A. Taube $\cdot$ M. Koenen $\cdot$ \\ B. Platzbecker • A. Cramer • A. Horrighs • \\ M. Lehtonen $\cdot$ N. Tennagels $\cdot$ J. Eckel
}

Received: 4 July 2008 / Accepted: 20 November 2008 / Published online: 17 December 2008

(C) Springer-Verlag 2008

\begin{abstract}
Aims/hypothesis Cannabinoid type 1 receptor (CB1R) antagonists such as rimonabant (Rim) represent a novel approach to treat obesity and related metabolic disorders. Recent data suggest that endocannabinoids are also produced by human adipocytes. Here we studied the potential involvement of endocannabinoids in the negative crosstalk between fat and muscle.

Methods The protein level of CB1R in human skeletal muscle cells (SkM) during differentiation was analysed using western blotting. SkM were treated with adipocyteconditioned medium (CM) or anandamide (AEA) in combination with the CB1R antagonists Rim or AM251, and insulin-stimulated Akt phosphorylation and glucose uptake were determined. Furthermore, signalling pathways of CB1R were investigated.

Results We revealed an increase of CB1R protein in SkM during differentiation. Twenty-four hour incubation of SkM with CM or AEA impaired insulin-stimulated Akt(Ser473)
\end{abstract}

Electronic supplementary material The online version of this article (doi:10.1007/s00125-008-1240-4) contains supplementary material, which is available to authorised users.

K. Eckardt $\cdot$ H. Sell $\cdot$ A. Taube $\cdot$ M. Koenen $\cdot$ B. Platzbecker $\cdot$

A. Cramer $\cdot$ A. Horrighs $\cdot$ J. Eckel $(\square)$

Institute of Clinical Biochemistry and Pathobiochemistry,

German Diabetes Center,

Auf'm Hennekamp 65,

40225 Duesseldorf, Germany

e-mail: eckel@uni-duesseldorf.de

M. Lehtonen

Department of Pharmaceutical Chemistry, University of Kuopio,

Kuopio, Finland

N. Tennagels

Sanofi-aventis,

Frankfurt, Germany phosphorylation by $60 \%$ and up to $40 \%$, respectively. Pretreatment of cells with Rim or AM251 reduced the effect of CM by about one-half, while the effect of AEA could be prevented completely. The reduction of insulinstimulated glucose uptake by $\mathrm{CM}$ was completely prevented by Rim. Short-time incubation with AEA activated extracellular regulated kinase $1 / 2$ and p38 mitogen-activated protein kinase, and impaired insulin-stimulated Akt (Ser473) phosphorylation, but had no effect on Akt (Thr308) and glycogen synthase kinase $3 \alpha / \beta$ phosphorylation. In addition, enhanced IRS-1 (Ser307) phosphorylation was observed.

Conclusions/interpretation Our results show that the CB1R system may play a role in the development of insulin resistance in human SkM. The results obtained with CM support the notion that adipocytes may secrete factors which are able to activate the CB1R. Furthermore, we identified two stress kinases in the signalling pathway of AEA and enhanced IRS-1(Ser307) phosphorylation, potentially underlying the development of insulin resistance.

Keywords Adipocyte-conditioned medium · Anandamide . Cannabinoid receptor type $1 \cdot$ Endocannabinoids .

Human skeletal muscle cells · Insulin resistance $\cdot$ Rimonabant

$\begin{array}{ll}\text { Abbreviations } \\ \text { AEA } & \text { anandamide } \\ \text { 2-AG } & \text { 2-arachidonoylglycerol } \\ \text { AMPK } \alpha 1 & \text { AMP-activated protein kinase alpha 1 } \\ \text { CB1R } & \text { cannabinoid type 1 receptor } \\ \text { CM } & \text { adipocyte-conditioned medium } \\ \text { DHEA } & \text { docosahexaenoyl ethanolamide } \\ \text { 2-DOG } & \text { 2-deoxy-D- }\left[{ }^{14} \text { C]glucose }\right. \\ \text { ECL } & \text { enhanced chemiluminescence } \\ \text { ERK1/2 } & \text { extracellular regulated kinase 1/2 }\end{array}$




$\begin{array}{ll}\text { FAAH } & \text { fatty acid amide hydrolase } \\ \text { GSK3 } & \text { glycogen synthase kinase } 3 \\ \text { HRP } & \text { horseradish peroxidase } \\ \text { JNK } & \text { c-jun N-terminal kinase } \\ \alpha \text { MEM } & \alpha \text {-modified Eagle's medium } \\ \text { mTOR } & \text { mammalian target of rapamycin } \\ \text { NFkB } & \text { nuclear factor kappaB } \\ \text { OEA } & \text { oleoyl ethanolamide } \\ \text { p38 MAPK } & \text { p38 mitogen-activated protein kinase } \\ \text { PDK } & \text { pyruvate dehydrogenase kinase } \\ \text { PEA } & \text { palmitoyl ethanolamide } \\ \text { Rim } & \text { rimonabant } \\ \text { SkM } & \text { skeletal muscle cells }\end{array}$

\section{Introduction}

The endocannabinoid system is a complex network involved in multiple physiological processes. Among other aspects, it has been shown to be important for control of energy homeostasis and body weight [1-3], drug addiction [4] and modulation of pain [5]. The endocannabinoid system comprises the cannabinoid receptors type 1 (CB1R) and 2, and their natural ligands called endocannabinoids, whereas anandamide (AEA) and 2-arachidonoylglycerol (2-AG) are the most intensively studied ligands. They act as retrograde messengers at presynaptic CB1R or target the receptors in an autocrine/paracrine manner [6]. Signals induced by the endocannabinoids are terminated very rapidly by a transporter-mediated uptake [7] and subsequent degradation. AEA is degraded by fatty acid amide hydrolase (FAAH) [8], while 2-AG is mainly hydrolysed by monoacylglyceride lipase [9].

Data from animal studies, and clinical studies in humans, have shown that in the obese state the endocannabinoid system is overactivated because of impaired energy balance. Genetically and diet-induced obese animal models show elevated levels of endocannabinoids in the hypothalamus and peripheral tissues [10-12]. In obese or hyperglycaemic type 2 diabetic patients, circulating levels of AEA and 2-AG are increased and elevated levels of 2-AG are found in visceral adipose tissue $[10,13,14]$. Furthermore, it is known from the $\mathrm{Cblr}^{--}$mouse model (up-todate symbol for mouse $\mathrm{Cblr}$ gene is $\mathrm{Cnrl}$ ) that pharmacological targeting of CB1R may be a promising approach for treating obesity and related metabolic disorders. $\mathrm{Cb}_{1 r^{-/}}$ mice are resistant to diet-induced obesity and remain lean on a high-fat diet without changes in metabolic or hormonal profiles $[11,15]$.

Obesity is one of the major components of the metabolic syndrome and a strong risk factor for the development of type 2 diabetes. It is associated with increased plasma levels of NEFAs and triacylglycerols, which contribute to insulin resistance in peripheral tissues such as skeletal muscle [16, 17]. Furthermore, adipose tissue is highly active in secreting a large number of factors such as cytokines and inflammation-related proteins $[18,19]$. Previous work in our laboratory has shown in vitro that adipocyte-conditioned medium $(\mathrm{CM})$ directly impairs insulin signalling in human myotubes $[20,21]$. Recently, the production of endocannabinoids by adipocytes and the involvement of endocannabinoids in the differentiation process of adipocytes has been shown [10, 22-24]. However, the impact of the endocannabinoid system on skeletal muscle metabolism has been investigated less intensively. One study analysed mRNA expression of genes regulating energy metabolism in skeletal muscle cells (SkM) after incubation with endocannabinoids [25]. Another study showed that in a genetically obese mouse model rimonabant (Rim) treatment increased basal glucose uptake in muscle [26]. To the best of our knowledge, so far no study has directly assessed whether a link between activation of skeletal muscle CB1R and the induction of insulin resistance exists. Therefore, we aimed to investigate the potential involvement of endocannabinoids in the negative crosstalk between fat tissue and skeletal muscle and associated signalling pathways.

\section{Methods}

Material Reagents for SDS-PAGE were supplied by GE Healthcare Bio-Sciences (Uppsala, Sweden) and Sigma (Munich, Germany). AEA and AM251 were from by Tocris Biosciences (Bristol, UK), Rim was kindly provided by Sanofi-aventis (Frankfurt, Germany). 2-Deoxy-D- $\left[{ }^{14} \mathrm{C}\right]$ glucose (2-DOG) and L- $\left[{ }^{14} \mathrm{C}\right]$ glucose were purchased from GE Healthcare (Uppsala, Sweden). Liquid scintillation Aqua safe 300 plus from Zinsser Analytic (Frankfurt, Germany) was used for glucose uptake assays. The phosphatase and protease inhibitor cocktail tablets were from Roche (Mannheim, Germany). To analyse the endocannabinoid concentrations, internal standards from Cayman Chemicals (Ann Arbor, MI, USA) were used. The commercially available antibodies used for immunoblotting are described in detail in the Electronic supplementary material (ESM) Table 1. The GLUT1 antibody was a gift from S. Sasson (Hebrew University, Jerusalem, Israel). Whole cell lysates from human hippocampus and human spinal cord were provided by Abcam (Cambridge, UK). The ELISA kit for phosphoIRS-1(Ser307) was purchased from Cell Signaling Technology (Massachusetts, USA). Culture media was supplied by Gibco (Berlin, Germany). Primary human SkM and the supplement pack for growth medium were obtained from PromoCell (Heidelberg, Germany). All other chemicals were of the highest analytical grade commercially available and were purchased from Sigma. 
Generation of $C M \mathrm{CM}$ was generated as described previously [27]. Briefly, human preadipocytes were isolated from adipose tissue samples obtained from subcutaneous fat of normal or moderately overweight women. The cells were cultured and differentiated for 15 days. The mature adipocytes were used to generate CM by incubation with $\alpha$-modified Eagle's medium ( $\alpha$ MEM) for $48 \mathrm{~h}$ followed by collection of the medium.

Culture of human SkM Primary human SkM of seven healthy donors (white, male, 9, 5 and 47 years [M9, M5, M47]; female, 10, 48, 49 and 37 years [F10, F48, F49, F37]) were supplied as proliferating myoblasts and cultured according to the protocol of PromoCell (Heidelberg, Germany). For an individual experiment, myoblasts were seeded into six well culture dishes and cultured in $\alpha \mathrm{MEM} /$ Ham's F-12 medium containing SkM growth medium supplement up to near confluence. The cells were then differentiated and fused by culture in $\alpha$ MEM for 6 days.

The differentiated cells were incubated with $\mathrm{CM}$ or $0.1-$ $10 \mu \mathrm{mol} / 1 \mathrm{AEA}$ for $24 \mathrm{~h}$ to induce insulin resistance. Afterwards, the cells were stimulated with $100 \mathrm{nmol} / 1$ insulin for $10 \mathrm{~min}$, lysed and processed as described below. To block the effects of CM and AEA, cells were preincubated with CB1R antagonists Rim $(1 \mu \mathrm{mol} / \mathrm{l})$ or AM251 $(10 \mu \mathrm{mol} / \mathrm{l})$ for $2 \mathrm{~h}$ before adding $\mathrm{CM}$ or AEA. To further analyse the kinetics of AEA-induced effects, SkM were incubated with $10 \mu \mathrm{mol} / \mathrm{l}$ AEA for different times from $5 \mathrm{~min}$ to $24 \mathrm{~h}$, stimulated with $100 \mathrm{nmol} / 1$ insulin, lysed and processed as described below.

Extraction and analysis of endocannabinoids CM samples were liquid-liquid extracted with methanol and chloroform to yield a methanol/chloroform/water ratio of 1:2:1 (by vol.). A reversed-phase column was used for separating analytes with an isocratic mobile phase. Stable isotopelabelled internal standards were included in the analysis, and detection was made with a triple quadrupole mass spectrometer using multiple reaction monitoring. Ionisation was performed with positive electrospray ionisation. Selectivity studies show that the liquid chromatography/MS/MS method was free from a matrix effect.

Glucose transport assay Differentiated SkM were preincubated with $1 \mu \mathrm{mol} / \mathrm{l} \mathrm{Rim}$ for $2 \mathrm{~h}$, then $\mathrm{CM}$ was added and the incubation was continued for $24 \mathrm{~h}$. To analyse the effect of AEA on glucose uptake, cells were treated with 1 or $10 \mu \mathrm{mol} / 1 \mathrm{AEA}$ for $24 \mathrm{~h}$. Afterwards, the cells were stimulated with $100 \mathrm{nmol} / \mathrm{l}$ insulin for $30 \mathrm{~min}$. Then, 2-DOG $(9.25 \mathrm{kBq} / \mathrm{ml}$ per well) was added and uptake was measured for $2 \mathrm{~h}$. The experiment was terminated by repeated washing with ice-cold $0.25 \mu \mathrm{mol} / 1$ cytochalasin $\mathrm{B}$ and lysis with $1 \mathrm{~mol} / \mathrm{l} \mathrm{NaOH}$. The radioactivity of the cell material was counted in a liquid scintillation counter (Beckman, Munich, Germany). Values were corrected for non-specific uptake, as measured after incubation with $\mathrm{L}-\left[{ }^{14} \mathrm{C}\right]$ glucose.

Immunoblotting For analysis of the protein level of CB1R, lysates from SkM of several donors at different days of differentiation were prepared, starting from myoblasts (day 0) to myotubes (day 6). For comparison and as a control, lysates of differentiated human adipocytes as well as commercially available whole cell lysates of human hippocampus and spinal cord were used. Lysates were prepared with ice-cold lysis buffer containing $50 \mathrm{mmol} / \mathrm{l}$ HEPES (pH 7.4), 1\% (vol./vol.) Triton X-100, phosphatase and protease inhibitor cocktail. After incubation for $2 \mathrm{~h}$ at $4^{\circ} \mathrm{C}$, the suspension was centrifuged at $10,000 \mathrm{~g}$ for $20 \mathrm{~min}$. Equal amounts of protein were loaded per sample, separated by SDS-PAGE and transferred to polyvinylidene fluoride membranes. The membranes were blocked in TRIS-buffered saline containing $0.1 \%$ (vol./vol.) Tween20 and $5 \%$ (wt/vol.) non-fat dry milk or $5 \%$ (wt/vol.) BSA and were then incubated overnight with the appropriate antibodies. After repeated washing, the membranes were incubated with horseradish peroxidase (HRP)-conjugated secondary antibody for $1 \mathrm{~h}$ at room temperature. Immobilon HRP substrate from Millipore (Billerica, MA, USA) was used for enhanced chemiluminescence (ECL) detection. The signals were visualised and evaluated on a LumiImager work station using image analysis software (Boehringer Mannheim, Mannheim, Germany).

Phosphorylation of IRS-1 To determine whether AEA influences the phosphorylation of IRS-1, an ELISA specific for IRS-1(Ser307) was used. Differentiated SkM were treated with $10 \mu \mathrm{mol} / \mathrm{l}$ AEA for $24 \mathrm{~h}$, stimulated with $100 \mathrm{nmol} / \mathrm{l}$ insulin and lysed. The lysates were sonicated on ice and centrifuged at $10,000 \mathrm{~g}$ for $10 \mathrm{~min}$ at $4^{\circ} \mathrm{C}$. The protein concentration of the supernatant fraction was quantified, and equal amounts per sample were added to each microwell. After completing the incubation procedure, the results were obtained by spectrophotometric determination.

Presentation of data and statistics Statistical analysis was carried out by ANOVA. All statistical analyses were done using StatView software (SAS Institute, Cary, NC, USA). A $p$ value $<0.05$ was considered to be statistically significant. Corresponding significance levels are indicated in the figures. Data are presented as means \pm SEM.

\section{Results}

Protein level of CBIR increases during differentiation of human $S k M$ We analysed the protein level of CB1R in 
human SkM of several donors during differentiation (Fig. 1) and detected a band at approximately $53 \mathrm{kDa}$ by western blotting, a molecular mass associated with a low glycosylation form of CB1R [13]. The protein level of the receptor increased during differentiation from myoblasts to myotubes and reached a level at day 6 comparable to that found in differentiated human adipocytes, at least in some muscle donors. Also, we observed differences in protein levels between the analysed SkM donors. Our results suggest that CB1R plays a relevant role in this peripheral tissue.

Partial prevention of CM-induced insulin resistance by CB1R antagonists Rim and AM251 To investigate whether activation of the cannabinoid system plays a role in the induction of insulin resistance by $\mathrm{CM}$, we aimed to block CB1R by incubation with the specific CB1R antagonists Rim or AM251, an analogue of Rim [28, 29]. Treatment of differentiated SkM with CM impaired insulin-stimulated Akt(Ser473) phosphorylation by $\sim 60 \%$, as shown in Fig. 2c, although insulin still produced a significant increase of $\operatorname{Akt}(\operatorname{Ser} 473)$ phosphorylation compared with basal. Pre-incubation with Rim or AM251 significantly reduced the inhibiting effect of $\mathrm{CM}$ on Akt activation to $\sim 20 \%$. Nevertheless, the pre-incubation could not completely abolish the effect of CM on Akt activation. This indicates that $\mathrm{CM}$ contains additional factors which take part in the induction of insulin resistance and do not stimulate the CB1R. Incubation with CM, Rim or AM251 did not alter the level of Akt protein (Fig. 2b).

\section{Rim prevents CM-induced impairment of insulin-stimulated} glucose uptake To further analyse the effect of $\mathrm{CM}$ combined with Rim at a level downstream of the signalling cascade, we assessed 2-DOG uptake. As shown in Fig. 3, insulin induced a $\sim 1.8$-fold increase of 2-DOG uptake under control conditions. Incubation of cells with $\mathrm{CM}$ for $24 \mathrm{~h}$ significantly impaired the insulin-stimulated glucose uptake to 1.5 -fold compared with basal without changing basal uptake rates. Treatment of SkM with Rim surprisingly resulted in a shift of 2-DOG uptake to a lower level because of significantly reduced basal uptake rates (Fig. 3). The
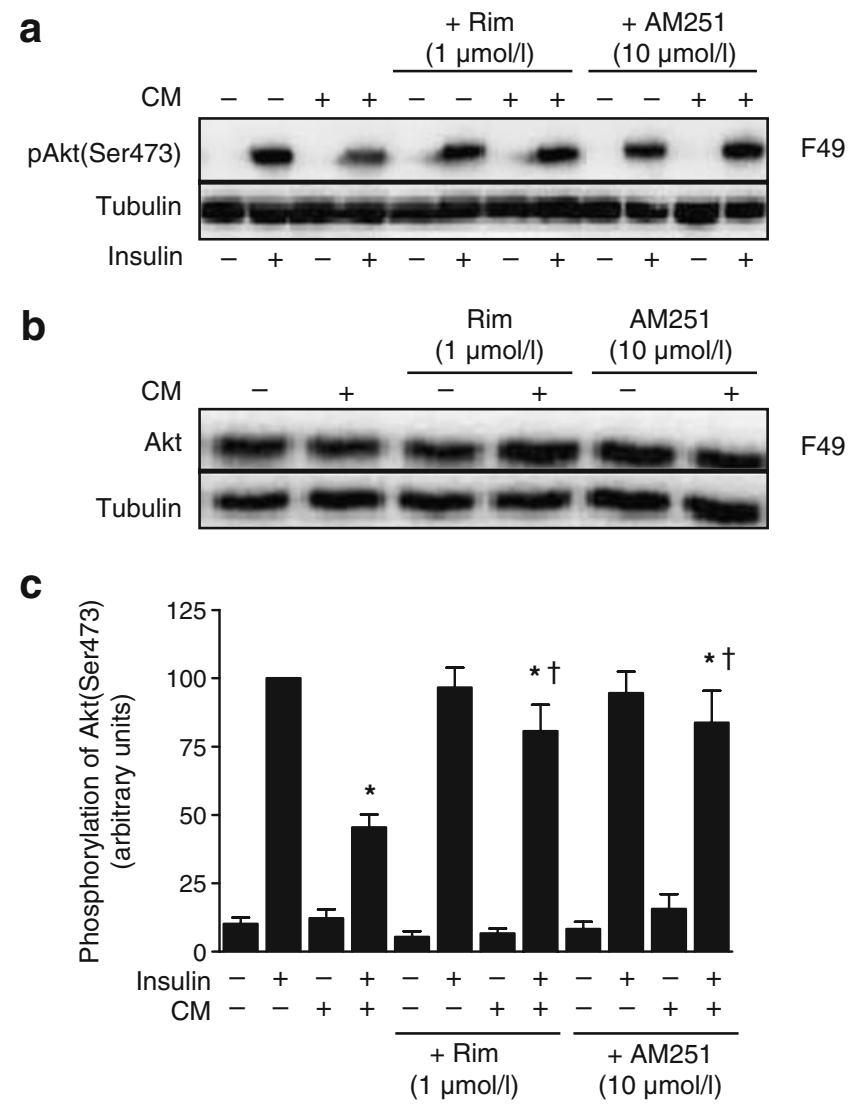

Fig. 2 Partial blocking of CM-induced insulin resistance by Rim and AM251. Differentiated SkM were pre-incubated with $1 \mu \mathrm{mol} / 1 \mathrm{Rim}$ or $10 \mu \mathrm{mol} / 1 \mathrm{AM} 251$ for $2 \mathrm{~h}$. Then, CM was added and incubation was continued for $24 \mathrm{~h}$. Afterwards cells were stimulated with $100 \mathrm{nmol} / \mathrm{l}$ insulin for $10 \mathrm{~min}$ and lysed. Whole cell lysates were resolved by SDS-PAGE, blotted and immunodetected with a phosphospecific Akt antibody using the ECL system. a Representative western blot of donor F49 showing Akt(Ser473) phosphorylation (p) after insulin stimulation. b Representative western blot of donor F49 showing protein levels of Akt after the indicated treatment. c Quantification of Akt(Ser473) phosphorylation after indicated treatments. All data were normalised to the level of $\alpha$-tubulin production and are expressed relative to the insulin-stimulated controls. Results represent mean values obtained from two different donors. Means \pm SEM, $n \geq 4$. ${ }^{*} p<0.05$ vs non-CM-treated insulin-stimulated controls. ${ }^{\dagger} p<0.05$ vs CM-treated insulin-stimulated controls

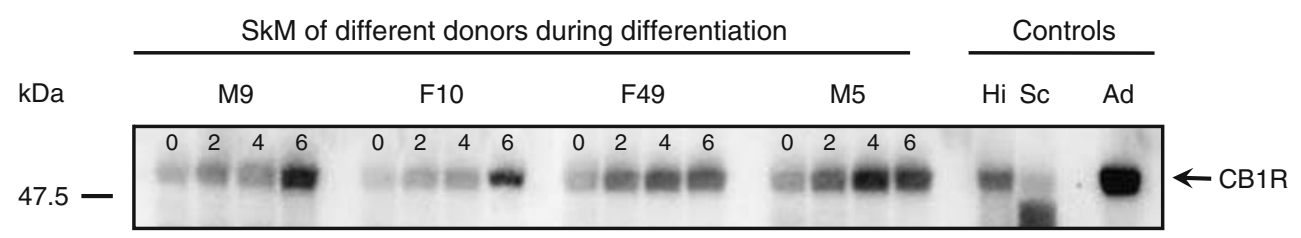

Fig. 1 Protein levels of CB1R in human SkM during differentiation. Ten micrograms of protein of whole cell lysates from SkM of the donors M9, F10, F49 and M5, and of human hippocampus (Hi), human spinal cord $(\mathrm{Sc})$ and differentiated human adipocytes (Ad) were resolved by SDS-PAGE, blotted and immunodetected with antiCB1R antibody using the ECL system. The numbers indicate the day of differentiation; day 0 represents myoblasts 


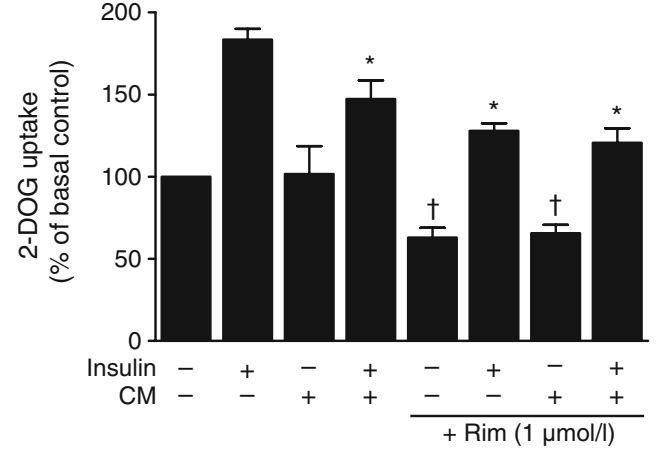

Fig. 3 Insulin-stimulated 2-DOG uptake. Differentiated SkM (donor F49) were pre-incubated with $1 \mu \mathrm{mol} / \mathrm{l}$ Rim for $2 \mathrm{~h}$. Then, CM was added and incubation was continued for $24 \mathrm{~h}$. Afterwards, cells were stimulated with $100 \mathrm{nmol} / \mathrm{l}$ insulin for $30 \mathrm{~min}$ and 2-DOG uptake was measured for $2 \mathrm{~h}$. The measured radioactivity of the cell lysates was corrected for non-specific uptake. The data are expressed relative to the basal glucose uptake of untreated controls. Means \pm SEM, $n \geq 3$. ${ }^{*} p<0.05$ vs non $\mathrm{CM}$-treated insulin-stimulated controls. ${ }^{\dagger} p<0.05$ vs non $\mathrm{CM}-$ treated basal controls

reason for this shift is currently not known, and analysis of GLUT1 and GLUT4 levels revealed no difference after incubation with Rim compared with controls (data not shown). Importantly, insulin stimulation caused a twofold increase in 2-DOG uptake in Rim-treated cells, which was not significantly affected by the presence of CM. These data indicate that Rim is able to prevent $\mathrm{CM}$-induced insulin resistance of glucose uptake in SkM.

CM contains endocannabinoids The ability of Rim to partially prevent CM-induced effects raises the question whether CM contains endocannabinoids. Therefore, we analysed $\mathrm{CM}$ obtained from different donors of adipocytes and detected AEA and 2-AG as well as palmitoyl ethanolamide (PEA), docosahexaenoyl ethanolamide (DHEA) and oleoyl ethanolamide OEA (Fig. 4). We measured concentrations of $0.63 \pm 0.1 \mathrm{pmol} / 1$ for AEA, $66.4 \pm 3.2 \mathrm{pmol} / \mathrm{l}$ for $2-\mathrm{AG}, 48.76 \pm 3.6 \mathrm{pmol} / \mathrm{l}$ for PEA, 1.13

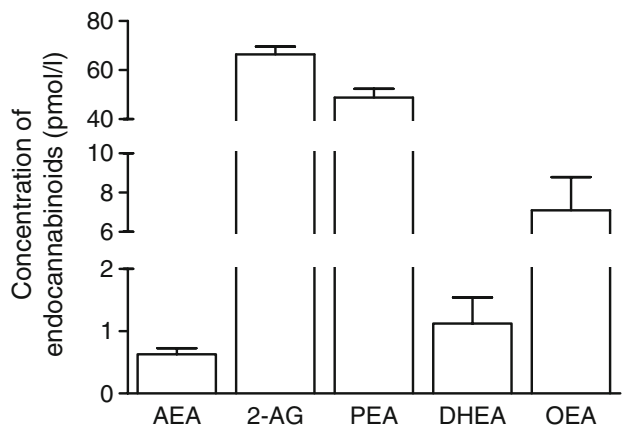

Fig. 4 Assessment of endocannabinoids in CM. CM was generated as described and analysed by liquid chromatography/MS/MS. Besides AEA and 2-AG, various AEA analogues were detected. Means \pm SEM, $n=3$
$0.4 \mathrm{pmol} / 1$ for DHEA and $7.09 \pm 1.7 \mathrm{pmol} / 1$ for OEA. In culture medium alone, which was used as background control, no endocannabinoids were detected.

Insulin resistance can be induced by the endogenous cannabinoid AEA To further investigate the role of CB1R in insulin resistance, we incubated differentiated SkM with the endocannabinoid AEA. As shown in Fig. 5a, c, $24 \mathrm{~h}$ incubation with AEA decreased the insulin-stimulated Akt (Ser473) phosphorylation in a concentration-dependent manner, with a maximal reduction by $\sim 40 \%$ when using

a

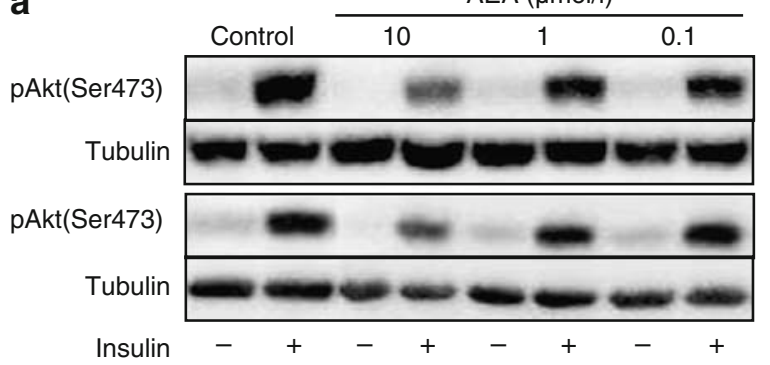

b

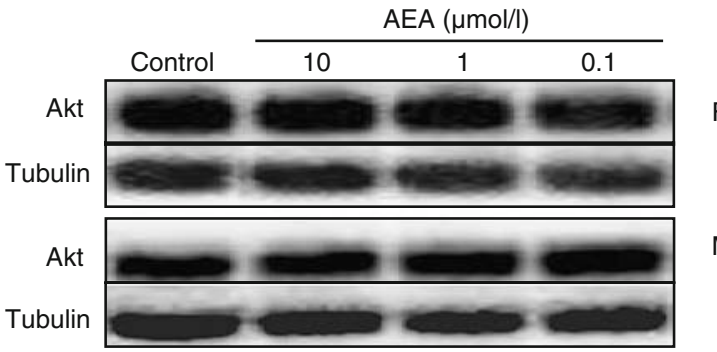

C

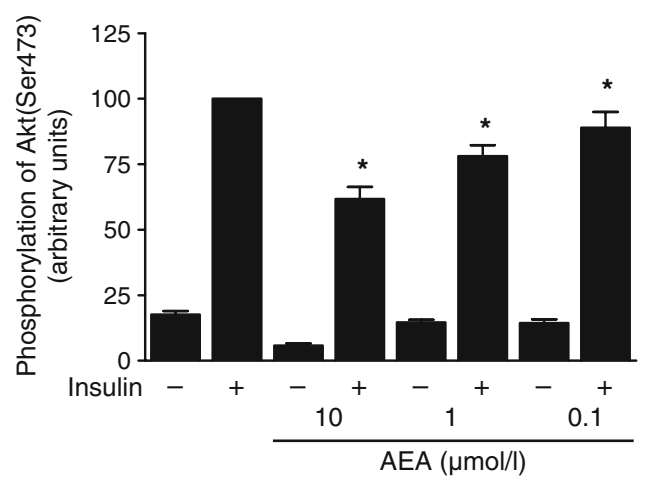

Fig. 5 Induction of insulin resistance by AEA. Differentiated SkM were incubated with $0.1-10 \mu \mathrm{mol} / 1$ AEA for $24 \mathrm{~h}$. Afterwards, cells were stimulated with $100 \mathrm{nmol} / 1$ insulin for $10 \mathrm{~min}$, lysed and processed as described in Fig. 2. a Representative western blots of donor F10 and M9 showing Akt(Ser473) phosphorylation (p) after insulin stimulation. b Representative western blots of donor F10 and M9 showing protein level of Akt. c Quantification of Akt(Ser473) phosphorylation after indicated treatments. All data were normalised to the level of $\alpha$-tubulin production and are expressed relative to the insulin-stimulated controls. Results represent mean values obtained from three different donors. Means $\pm \mathrm{SEM}, n \geq 12$. ${ }^{*} p<0.05$ vs insulinstimulated controls 
AEA at $10 \mu \mathrm{mol} / \mathrm{l}$. The Akt protein level was not changed at any concentration (Fig. 5b). Furthermore, incubation at $10 \mu \mathrm{mol} / \mathrm{l}$ AEA for $24 \mathrm{~h}$ significantly disturbed glucose uptake, as shown in Fig. 6. We observed a prominent increase of basal 2-DOG uptake, which was not accompanied by an increase of GLUT1 or GLUT4 protein abundance (data not shown). Stimulation with insulin did not result in a further significant increase of 2-DOG uptake under these conditions. To ensure that the effect of AEA on Akt activation was mediated by CB1R, we pre-incubated the cells with AM251. Treatment of SkM with $10 \mu \mathrm{mol} / 1$ AM251 alone changed neither insulin-stimulated Akt(Ser473) phosphorylation (Fig. 7a, c) nor levels of the enzyme (Fig. 7b). Incubation with $1 \mu \mathrm{mol} / \mathrm{l}$ AEA for $24 \mathrm{~h}$ reduced insulin-stimulated Akt(Ser473) phosphorylation significantly to $\sim 80 \%$ of controls, which was prevented by pre-incubation with AM251 for $2 \mathrm{~h}$ (Fig. 7).

AEA specifically inhibits Akt(Ser473) phosphorylation To gain insight into the kinetics of AEA-mediated effects on insulin signalling, we performed time-course experiments and analysed insulin-stimulated Akt(Ser473) and (Thr308) as well as glycogen synthase kinase $3(\mathrm{GSK} 3) \alpha / \beta$ phosphorylation. For this purpose, SkM were incubated with $10 \mu \mathrm{mol} / 1 \mathrm{AEA}$ for $20 \mathrm{~min}$ to $24 \mathrm{~h}$ followed by stimulation with $100 \mathrm{nmol} / \mathrm{l}$ insulin. After $1 \mathrm{~h}$ of AEA treatment we observed a significantly impaired Akt(Ser473) phosphorylation of $\sim 30 \%$ (Fig. 8a). This effect remained stable up to $6 \mathrm{~h}$, and was still visible after $24 \mathrm{~h}$ (Fig. 5). However, AEA treatment did not significantly disturb insulin-stimulated Akt(Thr308) phosphorylation (Fig. 8b). Also, no effect on insulin-stimulated GSK $3 \alpha / \beta$ phosphorylation was detected (data not shown).

AEA activates mitogen-activated protein kinase in SkM We were interested in analysing possible signalling pathways

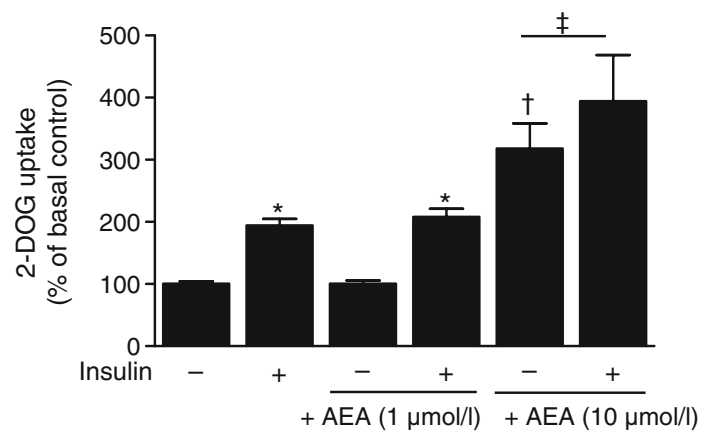

Fig. 6 AEA alters 2-DOG uptake. Differentiated SkM (donor F49) were incubated with 1 or $10 \mu \mathrm{mol} / 1$ AEA for $24 \mathrm{~h}$. Then 2-DOG uptake was performed as described. The data are expressed relative to the basal glucose uptake of untreated controls. The data are presented as means \pm SEM, $n \geq 3$. ${ }^{*} p<0.05$ vs basal uptake of each situation. ${ }^{\dagger} p<0.05$ vs basal controls. ${ }^{\dagger} p>0.05$

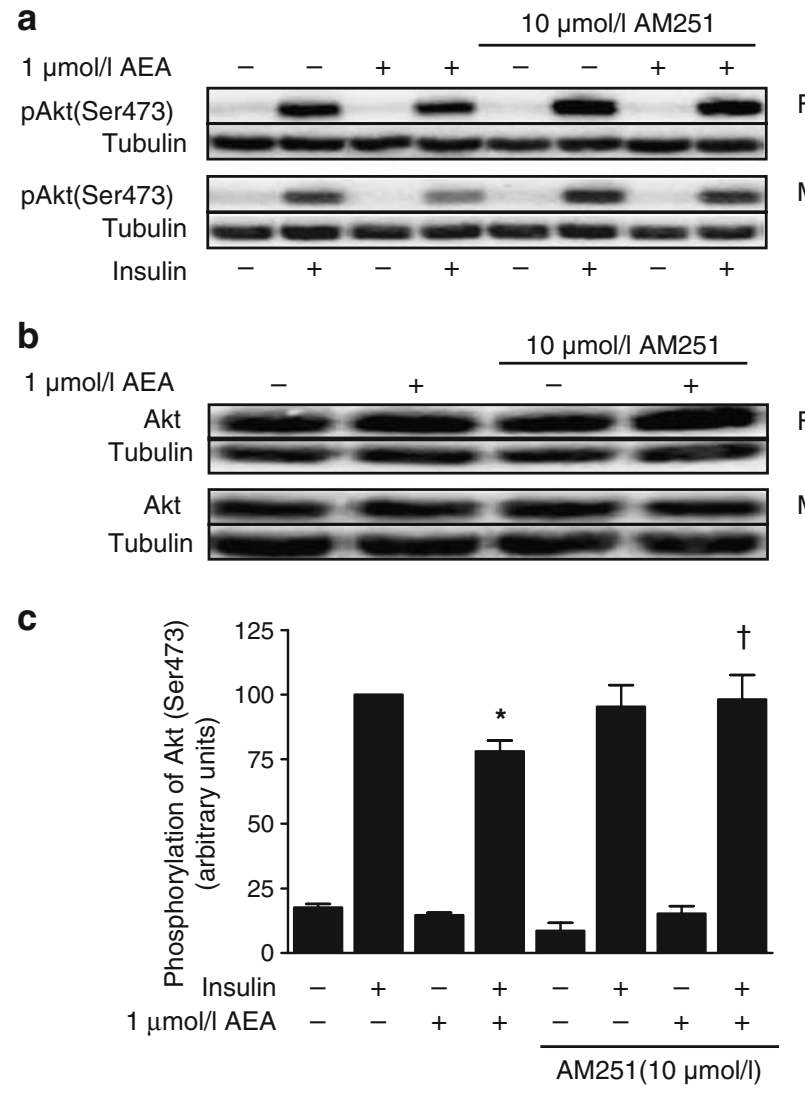

Fig. 7 AM251 prevents the effect of AEA. Differentiated SkM were pre-incubated with $10 \mu \mathrm{mol} / 1 \mathrm{AM} 251$ for $2 \mathrm{~h}$. Then $1 \mu \mathrm{mol} / \mathrm{l}$ AEA was added and incubation was continued for $24 \mathrm{~h}$. Afterwards, cells were stimulated with $100 \mathrm{nmol} / 1$ insulin for $10 \mathrm{~min}$, lysed and processed as described in Fig. 2. a Representative western blots of donor F10 and M9 showing Akt(Ser473) phosphorylation (p) after insulin stimulation. b Representative western blots of donor F10 and M9 showing protein level of Akt. c Quantification of Akt phosphorylation after indicated treatments. All data were normalised to the level of $\alpha$-tubulin production and are expressed relative to the insulinstimulated controls. Results represent mean values obtained from two different donors. Means \pm SEM, $n \geq 8$. ${ }^{*} p<0.05$ vs insulin-stimulated controls. ${ }^{\dagger} p<0.05$ vs AEA-treated insulin-stimulated situation

transmitting the effects of AEA and examined activation of extracellular regulated kinase 1/2 (ERK1/2), p38 mitogenactivated protein kinase (p38 MAPK), nuclear factor kappaB (NFKB) and c-jun N-terminal kinase (JNK). Treatment with $10 \mu \mathrm{mol} / \mathrm{l}$ AEA produced a pronounced phosphorylation of ERK1/2 as well as of p38 MAPK. As shown in Fig. 9a-c, a significant phosphorylation of ERK1/2 was detected after 5 min ( twofold of controls), which reached a peak at $10 \mathrm{~min}$ ( $\sim 3.8$-fold of controls). The phosphorylation rapidly declined thereafter and reached basal levels after $60 \mathrm{~min}$ (ERK1, Fig. 9b) and $30 \mathrm{~min}$ (ERK2, Fig. 9c), respectively. Activation of p38 MAPK was even more prominent, with a sevenfold higher phosphorylation compared with controls at 5 min (Fig. 9d, e). A peak was reached after $10 \mathrm{~min}$ followed by a slow decline 
a
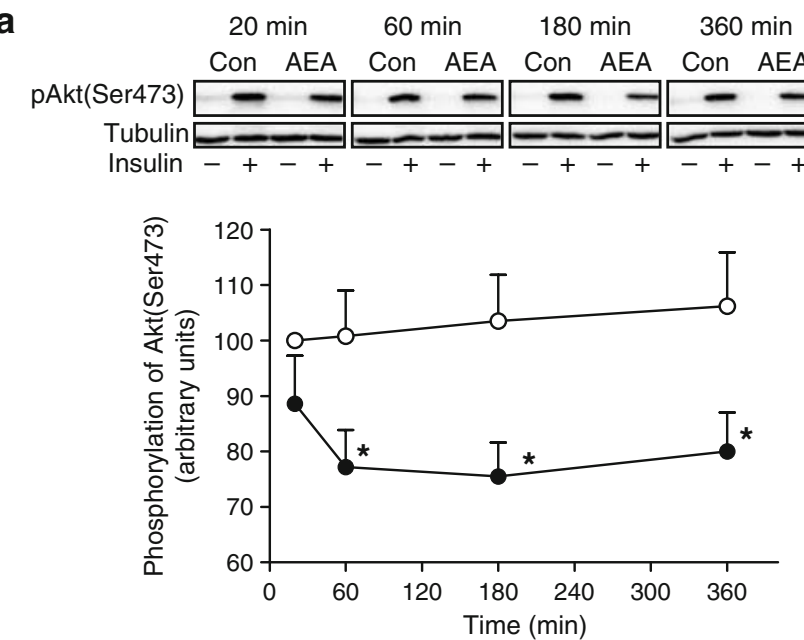

b
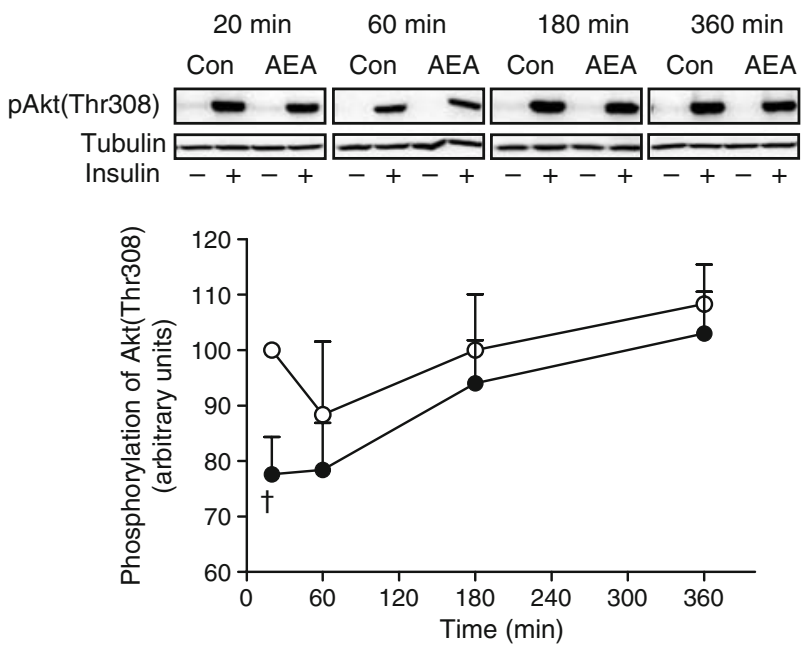

Fig. 8 AEA impairs insulin-stimulated Akt(Ser473) but not Akt (Thr308) phosphorylation (p). Differentiated SkM were incubated with $10 \mu \mathrm{mol} / 1$ AEA for 20-360 min followed by $10 \mathrm{~min}$ stimulation with insulin $(100 \mathrm{nmol} / \mathrm{l})$. Afterwards, cells were lysed and processed as described in Fig. 2. Representative western blots and quantification of insulin-stimulated Akt(Ser473) phosphorylation (a) and Akt (Thr308) phosphorylation (b). White circles, controls; black circles AEA. Presented are the insulin-stimulated phosphorylation levels. The data are expressed relative to the controls (con) at $20 \mathrm{~min}$. Results represent mean values obtained from three different donors. Means \pm SEM, $n \geq 3 .{ }^{*} p<0.05$ vs insulin-stimulated controls at the same timepoint. ${ }^{\dagger} p>0.05$

afterwards. In contrast to ERK1/2, phosphorylation of p38 MAPK was still significantly elevated compared with controls after $24 \mathrm{~h}$ AEA stimulation (Fig. 9f). However, we did not observe an activation of NFKB or JNK after incubation of SkM with AEA (data not shown).

AEA increases IRS-1(Ser307) phosphorylation In order to assess if AEA may disturb insulin signalling at an early point in the signalling cascade, we investigated whether AEA may affect IRS-1(Ser307) phosphorylation. This IRS-1 serine site is targeted by several kinases and known to negatively modulate insulin action. Under control conditions, insulin increases IRS-1(Ser307) phosphorylation - threefold over basal levels (Fig. 10). Remarkably, AEAtreated cells displayed a significant increase of basal and insulin-stimulated IRS-1(Ser307) phosphorylation (Fig. 10).

\section{Discussion}

The overactivation of the endocannabinoid system in human and animal obesity has been shown by various reports. Our CM-based model of insulin resistance mimics the crosstalk between adipocytes and SkM and induces insulin resistance at several levels [27]. We show here that pretreatment of SkM with CB1R antagonists prevents the perturbing effects of $\mathrm{CM}$. This observation prompted us to further investigate whether endocannabinoids might participate in the crosstalk between adipose tissue and skeletal muscle. Recently, the expression of cannabinoid receptors and production of enzymes for endocannabinoid synthesis and degradation were demonstrated in human adipose tissue [23], and another study showed the production of 2-AG and AEA by isolated mature adipocytes [22]. We demonstrate here that $\mathrm{CM}$ generated by human adipocytes contains endocannabinoids such as AEA and 2-AG, meaning that adipocytes not only produce but also secrete these compounds. The measured concentrations are very low compared with what is usually found in serum of patients or what we used in our in vitro experiments. However, besides AEA and 2-AG, we also measured PEA, DHEA and OEA. As for PEA, which does not bind to CB1R and is known for its anti-inflammatory effects [30], one possible mechanism may be the potentiation of AEA effects by competing with AEA for FAAH-mediated degradation [22]. Additionally, the ability of CB1R antagonists to partially prevent effects of $\mathrm{CM}$ make it reasonable to assume that activation of CB1R plays a role in the induction of SkM insulin resistance by $\mathrm{CM}$. We suggest that synergistic mechanisms may be involved because AEA itself in a concentration $<1 \mathrm{pmol} / 1$ will probably not have an effect on basal or insulin-stimulated signalling events in in vitro experiments. $\mathrm{CM}$ is a complex mixture of various factors secreted by adipocytes, and it is most likely that additional mechanisms besides CB1R activation are involved in the induction of insulin resistance. For example, previous reports from our laboratory have shown that $\mathrm{CM}$ impairs insulin-stimulated phosphorylation of GSK $3 \alpha / \beta$ in SkM [27], an effect we did not observe when treating SkM with AEA alone. However, the issue of endocannabinoid concentrations in vivo is more difficult to consider. Although analysis of human serum revealed AEA concen- 
a

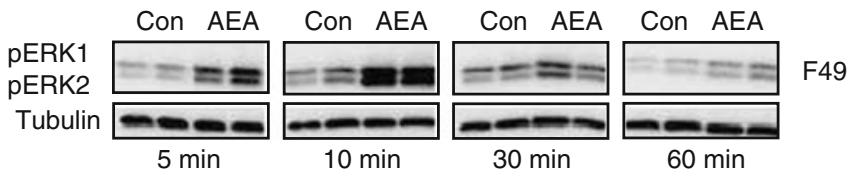

b

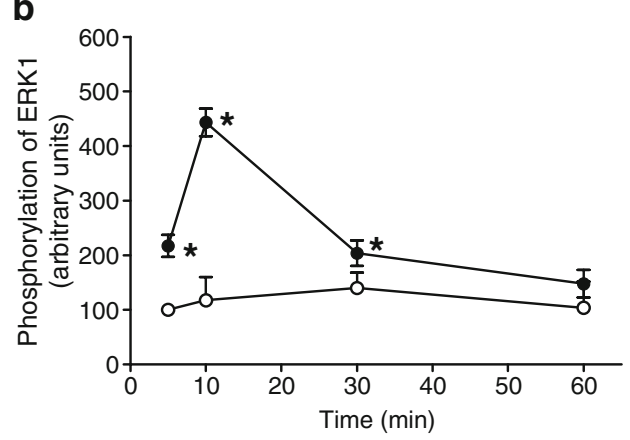

d

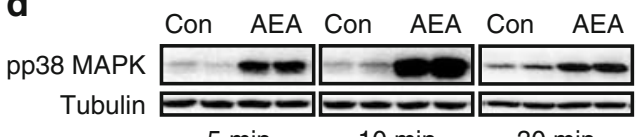

$5 \mathrm{~min}$

$10 \mathrm{~min}$

$30 \mathrm{~min}$

e

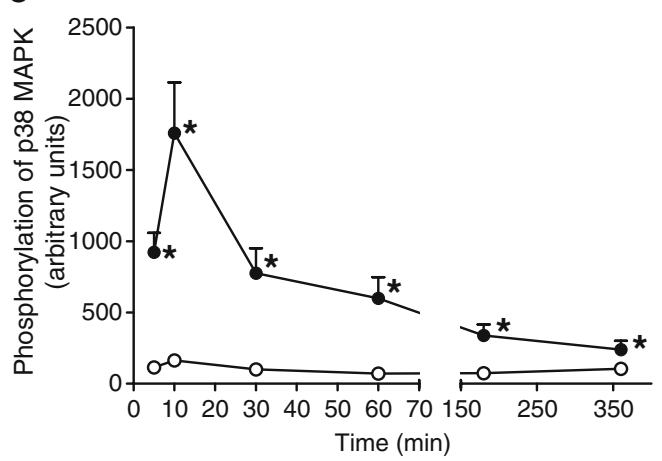

Fig. 9 Activation of ERK1/2 and p38 MAPK in response to AEA treatment. Differentiated SkM were incubated with $10 \mu \mathrm{mol} / 1$ AEA for the indicated times. Afterwards, cells were lysed and processed as described in Fig. 2, using specific phospho-antibodies. a Representative western blots of donor F49 showing phosphorylation (p) of ERK1/2 induced by stimulation with AEA. Quantification of ERK1 (b) and ERK2 (c) phosphorylation after treatment with AEA for 5-60 min (white circles, controls; black circles, AEA). The data are expressed relative to the phosphorylation level of controls (con) after $5 \mathrm{~min}$. d Representative western blots of donor W37 showing phosphorylation

trations between 2 and $2.7 \mathrm{pmol} / \mathrm{ml}[13,31]$, the concentrations in tissue may be much higher, since intramuscular adipocytes have direct contact to SkM and the interstitial space between adjacent cells is rather small. Taken together, our data show that adipose tissue is able to produce and secrete endogenous cannabinoids that may take part in the negative crosstalk between fat and muscle tissue leading to insulin resistance.

We show here that Rim is able to prevent the CMmediated decrease in insulin-stimulated glucose uptake.
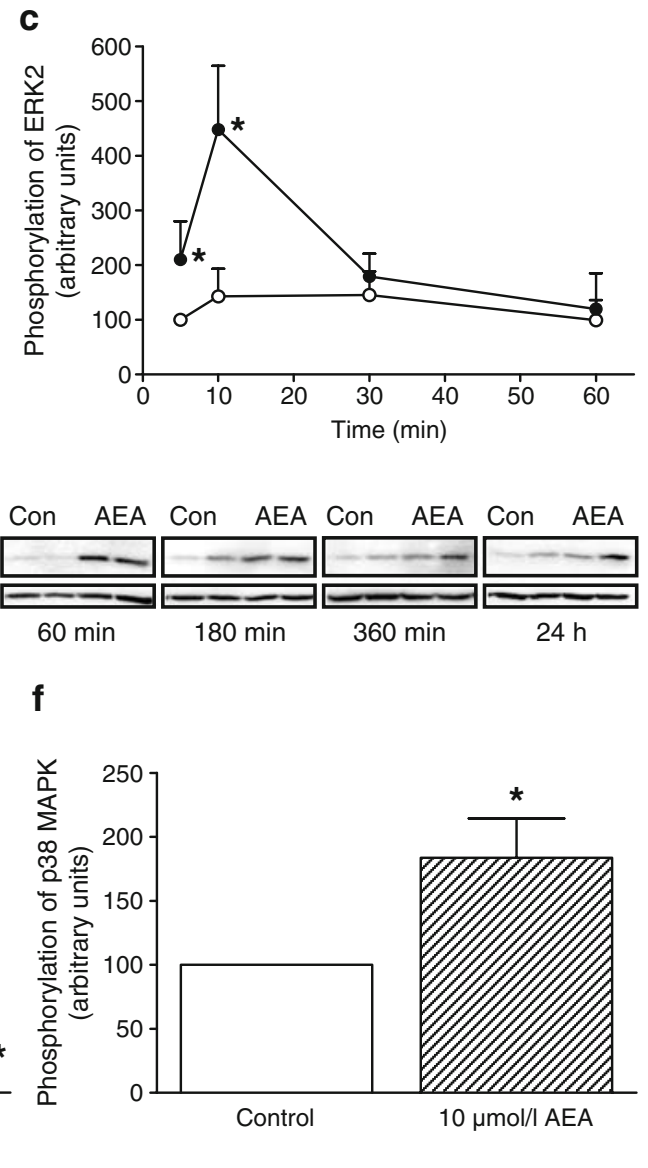

of p38 MAPK induced by stimulation with AEA. e Quantification of phosphorylated p38 MAPK (pp38 MAPK) after treatment with AEA for 30-360 min. The data are expressed relative to the phosphorylation level of controls after $30 \mathrm{~min}$. f Quantification of p38 MAPK phosphorylation after $24 \mathrm{~h}$ treatment with AEA. All data were normalised to the level of $\alpha$-tubulin production. Results represent mean values obtained from three different donors. Means \pm SEM, $n \geq 5$ for $\mathrm{pERK} 1 / 2(\mathbf{b}, \mathbf{c}), n \geq 3$ for pp38 MAPK (d, e). ${ }^{*} p<0.05$ vs $5 \mathrm{~min}$ controls $(\mathbf{b}, \mathbf{c})$, vs $30 \mathrm{~min}$ controls (e) and vs $24 \mathrm{~h}$ controls (f)

However, an unexpected finding was the decrease in basal 2-DOG uptake upon Rim treatment which can not be explained by altered GLUT1 or GLUT4 levels. One study [23] also reported a tendency for reduced basal and insulinstimulated glucose uptake in Rim-treated human adipoctyes compared with controls. This difference did not reach significance but is consistent with our data. On the other hand, our results seem to be in contrast to another study [26], which measured an increase of basal glucose uptake in isolated soleus muscle of $\mathrm{Lep}^{o b o b}$ mice after treating the 


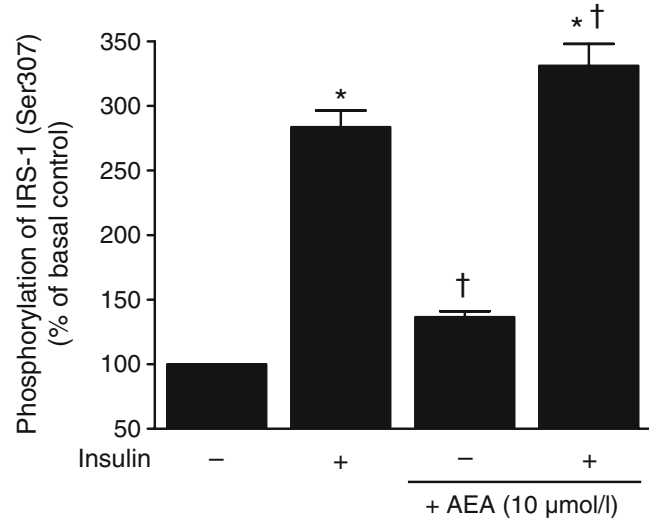

Fig. 10 AEA increases IRS-1(Ser307) phosphorylation. Differentiated SkM were incubated with $10 \mu \mathrm{mol} / \mathrm{l}$ AEA for $24 \mathrm{~h}$. Afterwards, cells were stimulated with $100 \mathrm{nmol} / 1$ insulin for $10 \mathrm{~min}$, lysed and used for IRS-1(Ser307)-specific ELISA. The data are expressed relative to basal level of IRS-1(Ser307) in controls. Results represent mean values obtained from two different donors. Means \pm SEM, $n \geq 3$. ${ }^{*} p<0.05$ vs basal phosphorylation in each situation. ${ }^{\dagger} p<0.05$ vs basal and insulin-stimulated controls, respectively

animals with Rim for 1 week. However, this experimental approach was indirect and did not analyse the direct effect of Rim on skeletal muscle since the tissue is also affected by physiological changes which may be secondary to Rim. It has been shown previously that Rim increases adiponectin expression in adipose tissue of obese rats [32], and circulating adiponectin is known to promote glucose uptake in skeletal muscle [33]. Very recently, it was reported that the improvement of insulin sensitivity in diet-induced obese mice by Rim treatment indeed requires adiponectin, while the effect on body weight was independent of adiponectin [34]. Therefore, the observed change of basal uptake observed in Rim-treated $L e p^{o b o b}$ mice may be caused by secondary rather than primary effects of Rim. Unfortunately, insulin-stimulated glucose uptake was not analysed, which is interesting in the light of possibly improved insulin sensitivity caused by Rim. In our study we used human SkM to directly investigate the impact of CB1R antagonists on CM-induced impairment of insulin-stimulated processes such as Akt phosphorylation and glucose uptake. We show here that pretreatment of SkM with CB1R antagonists prevents the negative effects of $\mathrm{CM}$ on insulinstimulated glucose uptake, supporting the notion that CB1R antagonists may exert beneficial effects in the periphery $[32,35,36]$, including skeletal muscle. Additional evidence for the benefit of CB1R antagonists are provided by a report of modified expression of genes involved in regulating the energy metabolism in SkM in response to AM251 [25]. The changes in pyruvate dehydrogenase kinase (PDK) 4 and AMP-activated protein kinase alpha 1 $(\mathrm{AMPK} \alpha 1)$ levels are consistent with increased glucose uptake and use and an overall increase in metabolic capacity. Furthermore, Rim could antagonise the effects of endocannabinoid action observed in adipocytes such as increased glucose uptake and GLUT4 translocation [23].

In obesity, the levels of serum and tissue endocannabinoids are known to be elevated. In obese patients with type 2 diabetes the circulating levels of AEA and 2-AG are increased two- to threefold [10]. Therefore, we used AEA for stimulation of SkM CB1R and found a concentrationdependent impairment of insulin-stimulated Akt(Ser473) phosphorylation. This effect could be completely abolished by pre-incubation with the CB1R antagonist AM251. Additionally, we demonstrate here that a high dose of AEA exerts a strong effect on basal glucose uptake. The observed increase may be related to activation of AMPK (K. Eckardt, unpublished data), which is known to be involved in the regulation of glucose uptake. Stimulation with insulin did not further increase 2-DOG uptake, indicating a state of insulin resistance.

Regarding possible mechanisms of AEA-induced insulin resistance, we analysed IRS-1 phosphorylation and observed an enhanced phosphorylation of IRS-1(Ser307) upon AEA treatment. This site is involved in negative regulation of the insulin signal, and it is known to be phosphorylated by several kinases, including inhibitory kappa B kinase-beta and JNK, thus leading to insulin resistance [37, 38]. Our results indicate that AEA causes an interference with insulin signalling at an early point in the cascade by enhancing IRS-1(Ser307) phosphorylation. Downstream of IRS-1, insulin-stimulated Akt(Ser473) phosphorylation was impaired with no effect on Akt (Thr308). Both sites are phosphorylated by distinct kinases, mammalian target of rapamycin (mTOR) C2 [39, 40] and PDK1 [41], respectively. Based on analysis of Akt mutants it is assumed that phosphorylation at both Thr308 and Ser473 is critical to generate a high level of Akt activity [42]. However, recent studies conducted in cells with ablated mTORC2 activity revealed that many targets of Akt including GSK3 are still phosphorylated despite reduced Akt activity, whereas other targets are not [43, 44]. Therefore, it may be speculated that AEA could affect mTORC2 and thus may impair insulin-stimulated Akt (Ser473) phosphorylation; however, direct evidence for this assumption is still lacking. While GSK3 phosphorylation is not disturbed, other downstream targets of Akt involved in glucose uptake regulation may be affected, since we observe disturbed insulin-stimulated glucose uptake. Thus, our data show that CB1R signalling in skeletal muscle only partly and very selectively interferes with the metabolic actions of insulin.

Another hint to possible mechanisms of AEA action are provided by phosphorylation of ERK1/2 and p38 MAPK. While ERK1/2 activation is detectable for $1 \mathrm{~h}$ of AEA treatment and declines afterwards to basal levels, the activation of p38 MAPK remains elevated for up to $24 \mathrm{~h}$. 
Both kinases are known to play a role in the development of insulin resistance. The involvement of ERK1/2 in the induction of insulin resistance by monocyte chemoattractant protein-1 (MCP-1) was shown by Sell et al. [27], who used the ERK1/2 specific inhibitor PD98059 and were therefore able to prevent MCP-1-induced insulin resistance. The involvement of p38 MAPK in TNF- $\alpha$-induced insulin resistance is described for SkM [45] and endothelial cells [46]. Additionally, Koistinen et al. [47] reported aberrant p38 MAPK signalling in skeletal muscle of type 2 diabetic patients. Our results also show that JNK and the proinflammatory NFKB signalling pathway seem not to be involved in the pathway of AEA-induced insulin resistance.

In summary, we demonstrate here for the first time that activation of CB1R could play a role in the induction of insulin resistance in human skeletal muscle. While AEA signalling is independent of JNK and the NFKB pathway, the activation of ERK1/2 and p38 MAPK are probably involved in this process. Treatment of SkM with AEA increases IRS-1(Ser307) phosphorylation, thus disturbing insulin signalling at an early point in the cascade. Analysis of $\mathrm{CM}$, which is a complex mixture of various factors secreted by adipocytes, revealed the presence of endocannabinoids, and the results obtained with CM in combination with CB1R antagonists support the notion that adipocytes secrete factors which are able to activate the CB1R. Hereby, our results add an additional component to the complex mechanisms that lead to the development of insulin resistance in skeletal muscle.

Acknowledgements This work was supported by the Ministerium für Wissenschaft und Forschung des Landes Nordrhein-Westfalen (Ministry of Science and Research of the State of North RhineWestphalia), the Bundesministerium für Gesundheit (Federal Ministry of Health), the Commission of the European Communities (Collaborative Project ADAPT, Contract No. HEALTH-F2-2008-201100), European Union COST Action BM0602 and the Jühling Foundation. The secretarial assistance of B. Hurow is gratefully acknowledged.

Duality of interest N. Tennagels is employee of Sanofi-aventis (Frankfurt, Germany). All other authors declare that there is no duality of interest associated with this manuscript.

\section{References}

1. Matias I, Di Marzo V (2007) Endocannabinoids and the control of energy balance. Trends Endocrinol Metab 18:27-37

2. Cota D, Marsicano G, Tschop M et al (2003) The endogenous cannabinoid system affects energy balance via central orexigenic drive and peripheral lipogenesis. J Clin Invest 112:423-431

3. Di Marzo V, Matias I (2005) Endocannabinoid control of food intake and energy balance. Nat Neurosci 8:585-589

4. Maldonado R, Valverde O, Berrendero F (2006) Involvement of the endocannabinoid system in drug addiction. Trends Neurosci 29:225-232
5. Pertwee RG (2001) Cannabinoid receptors and pain. Prog Neurobiol 63:569-611

6. de Fonseca R, Del AI, Bermudez-Silva FJ, Bilbao A, Cippitelli A, Navarro $M$ (2005) The endocannabinoid system: physiology and pharmacology. Alcohol Alcohol 40:2-14

7. Beltramo M, Stella N, Calignano A, Lin SY, Makriyannis A, Piomelli D (1997) Functional role of high-affinity anandamide transport, as revealed by selective inhibition. Science 277:1094-1097

8. Cravatt BF, Giang DK, Mayfield SP, Boger DL, Lerner RA, Gilula NB (1996) Molecular characterization of an enzyme that degrades neuromodulatory fatty-acid amides. Nature 384:83-87

9. Dinh TP, Carpenter D, Leslie FM et al (2002) Brain monoglyceride lipase participating in endocannabinoid inactivation. Proc Natl Acad Sci U S A 99:10819-10824

10. Matias I, Gonthier MP, Orlando P et al (2006) Regulation, function, and dysregulation of endocannabinoids in models of adipose and beta-pancreatic cells and in obesity and hyperglycemia. J Clin Endocrinol Metab 91:3171-3180

11. Osei-Hyiaman D, DePetrillo M, Pacher P et al (2005) Endocannabinoid activation at hepatic CB1 receptors stimulates fatty acid synthesis and contributes to diet-induced obesity. J Clin Invest 115:1298-1305

12. Di Marzo V, Goparaju SK, Wang L et al (2001) Leptin-regulated endocannabinoids are involved in maintaining food intake. Nature 410:822-825

13. Engeli S, Bohnke J, Feldpausch M et al (2005) Activation of the peripheral endocannabinoid system in human obesity. Diabetes $54: 2838-2843$

14. Bluher M, Engeli S, Kloting N et al (2006) Dysregulation of the peripheral and adipose tissue endocannabinoid system in human abdominal obesity. Diabetes 55:3053-3060

15. Ravinet-Trillou C, Delgorge C, Menet C, Arnone M, Soubrie P (2004) CB1 cannabinoid receptor knockout in mice leads to leanness, resistance to diet-induced obesity and enhanced leptin sensitivity. Int J Obes Relat Metab Disord 28:640-648

16. Dresner A, Laurent D, Marcucci M et al (1999) Effects of free fatty acids on glucose transport and IRS-1-associated phosphatidylinositol 3-kinase activity. J Clin Invest 103:253-259

17. Griffin ME, Marcucci MJ, Cline GW et al (1999) Free fatty acidinduced insulin resistance is associated with activation of protein kinase $\mathrm{C}$ theta and alterations in the insulin signaling cascade. Diabetes 48:1270-1274

18. Trayhurn P, Wood IS (2005) Signalling role of adipose tissue: adipokines and inflammation in obesity. Biochem Soc Trans 33:1078-1081

19. Sell H, Eckel J, Dietze-Schroeder D (2006) Pathways leading to muscle insulin resistance: the muscle-fat connection. Arch Physiol Biochem 112:105-113

20. Dietze D, Koenen M, Rohrig K, Horikoshi H, Hauner H, Eckel J (2002) Impairment of insulin signaling in human skeletal muscle cells by co-culture with human adipocytes. Diabetes 51:23692376

21. Dietze D, Ramrath S, Ritzeler O, Tennagels N, Hauner H, Eckel J (2004) Inhibitor kappaB kinase is involved in the paracrine crosstalk between human fat and muscle cells. Int J Obes Relat Metab Disord 28:985-992

22. Gonthier MP, Hoareau L, Festy F et al (2007) Identification of endocannabinoids and related compounds in human fat cells. Obesity (Silver Spring) 15:837-845

23. Pagano C, Pilon C, Calcagno A et al (2007) The endogenous cannabinoid system stimulates glucose uptake in human fat cells via PI3-kinase and calcium-dependent mechanisms. J Clin Endocrinol Metab 92:4810-4819

24. Gasperi V, Fezza F, Pasquariello N et al (2007) Endocannabinoids in adipocytes during differentiation and their role in glucose uptake. Cell Mol Life Sci 64:219-229 
25. Cavuoto P, McAinch AJ, Hatzinikolas G, Cameron-Smith D, Wittert GA (2007) Effects of cannabinoid receptors on skeletal muscle oxidative pathways. Mol Cell Endocrinol 267:63-69

26. Liu YL, Connoley IP, Wilson CA, Stock MJ (2005) Effects of the cannabinoid CB1 receptor antagonist SR141716 on oxygen consumption and soleus muscle glucose uptake in Lep(ob)/Lep (ob) mice. Int J Obes (Lond) 29:183-187

27. Sell H, Dietze-Schroeder D, Kaiser U, Eckel J (2006) Monocyte chemotactic protein-1 is a potential player in the negative crosstalk between adipose tissue and skeletal muscle. Endocrinology $147: 2458-2467$

28. Pertwee RG (2005) Inverse agonism and neutral antagonism at cannabinoid CB1 receptors. Life Sci 76:1307-1324

29. Gatley SJ, Gifford AN, Volkow ND, Lan R, Makriyannis A (1996) 123I-labeled AM251: a radioiodinated ligand which binds in vivo to mouse brain cannabinoid CB1 receptors. Eur J Pharmacol 307:331-338

30. Lo Verme J, Fu J, Astarita G et al (2005) The nuclear receptor peroxisome proliferator-activated receptor-alpha mediates the antiinflammatory actions of palmitoylethanolamide. Mol Pharmacol 67:15-19

31. Engeli S, Heusser K, Janke J et al (2008) Peripheral endocannabinoid system activity in patients treated with sibutramine. Obesity (Silver Spring) 16:1135-1137

32. Bensaid M, Gary-Bobo M, Esclangon A et al (2003) The cannabinoid CB1 receptor antagonist SR141716 increases Acrp30 mRNA expression in adipose tissue of obese fa/fa rats and in cultured adipocyte cells. Mol Pharmacol 63:908-914

33. Dyck DJ, Heigenhauser GJ, Bruce CR (2006) The role of adipokines as regulators of skeletal muscle fatty acid metabolism and insulin sensitivity. Acta Physiol (Oxf) 186:5-16

34. Migrenne S, Lacombe A, Lefevre AL et al (2008) Adiponectin is required to mediate rimonabant-induced improvements of insulin sensitivity but not body weight loss in diet-induced obese mice. Diabetes 57(Suppl 1):A31 (Abstract)

35. Gary-Bobo M, Elachouri G, Gallas JF et al (2007) Rimonabant reduces obesity-associated hepatic steatosis and features of metabolic syndrome in obese Zucker fa/fa rats. Hepatology 46:122-129

36. Schafer A, Pfrang J, Neumuller J, Fiedler S, Ertl G, Bauersachs J (2008) The cannabinoid receptor-1 antagonist rimonabant inhibits platelet activation and reduces pro-inflammatory chemokines and leukocytes in Zucker rats. Br J Pharmacol 154:1047-1054

37. Gao Z, Hwang D, Bataille F et al (2002) Serine phosphorylation of insulin receptor substrate 1 by inhibitor kappa B kinase complex. J Biol Chem 277:48115-48121

38. Aguirre V, Werner ED, Giraud J, Lee YH, Shoelson SE, White MF (2002) Phosphorylation of Ser307 in insulin receptor substrate-1 blocks interactions with the insulin receptor and inhibits insulin action. J Biol Chem 277:1531-1537

39. Hresko RC, Mueckler M (2005) mTOR.RICTOR is the Ser473 kinase for Akt/protein kinase B in 3T3-L1 adipocytes. J Biol Chem 280:40406-40416

40. Sarbassov DD, Guertin DA, Ali SM, Sabatini DM (2005) Phosphorylation and regulation of $\mathrm{Akt} / \mathrm{PKB}$ by the rictor-mTOR complex. Science 307:1098-1101

41. Alessi DR, James SR, Downes CP et al (1997) Characterization of a 3-phosphoinositide-dependent protein kinase which phosphorylates and activates protein kinase Balpha. Curr Biol 7:261269

42. Alessi DR, Andjelkovic M, Caudwell B et al (1996) Mechanism of activation of protein kinase B by insulin and IGF-1. EMBO J 15:6541-6551

43. Jacinto E, Facchinetti V, Liu D et al (2006) SIN1/MIP1 maintains rictor-mTOR complex integrity and regulates Akt phosphorylation and substrate specificity. Cell 127:125-137

44. Guertin DA, Stevens DM, Thoreen CC et al (2006) Ablation in mice of the mTORC components raptor, rictor, or mLST8 reveals that $\mathrm{mTORC} 2$ is required for signaling to Akt-FOXO and PKcalpha, but not S6K1. Dev Cell 11:859-871

45. De Alvaro C, Teruel T, Hernandez R, Lorenzo M (2004) Tumor necrosis factor alpha produces insulin resistance in skeletal muscle by activation of inhibitor kappaB kinase in a p38 MAPKdependent manner. J Biol Chem 279:17070-17078

46. Li G, Barrett EJ, Barrett MO, Cao W, Liu Z (2007) Tumor necrosis factor-alpha induces insulin resistance in endothelial cells via a p38 mitogen-activated protein kinase-dependent pathway. Endocrinology 148:3356-3363

47. Koistinen HA, Chibalin AV, Zierath JR (2003) Aberrant p38 mitogen-activated protein kinase signalling in skeletal muscle from Type 2 diabetic patients. Diabetologia 46:1324-1328 